TẠP CHÍ KHOA HỌC ĐẠI HỌC TÂN TRÀO

ISSN: 2354 - 1431

http://tckh.daihoctantrao.edu.vn/

\title{
Nâng cao hiệu quả huấn luyện thể lực chuyên môn của nam vận động viên wushu lứa tuổi 13-14 tỉnh Tuyên Quang
}

\author{
Trần Vũ Phuơng ${ }^{a *}$ Luc Hung Quốc ${ }^{a}$ \\ ${ }^{a}$ Trưòng Đại học Tân trào \\ *Email:phuongdhtt@gmail.com
}

\section{Thông tin bài viết}

Ngày nhận bài:

27/11/2018

Ngày duyệt đăng:

$10 / 12 / 2018$

Tù khoá:

Biện pháp; nâng cao hiệu quả; thể lực chuyên môn; huấn luyện viên, nam vận động viên Wushu 13-14 tuổi.

\section{Tóm tắt}

Để nâng cao công tác huấn luyện thể lực cho nam vận động viên (VĐV) đội tuyển Wushu lứa tuổi 13-14 tỉnh Tuyên Quang, đề tài đã lựa chọn được 3 nhóm biện pháp. Ba nhóm biện pháp này khẳng định được tính hiệu quả trong quá trình huấn luyện thể lực của nam VĐV Wushu lứa tuổi 13-14 tỉnh Tuyên Quang.

\section{1. Đặt vấn đề}

Trung tâm Huấn luyện Thể dục thể thao (TDTT) tỉnh Tuyên Quang là cái nôi đào tạo vận động viên (VĐV) trẻ để vươn tới thể thao đạt thành tích cao trong khu vực, đặc biệt phải kể đến thành tích của những môn Thể thao như: Pencat Silat, Wushu, Bóng đá, Cầu mây,... Những năm gần đây tỉnh Tuyên Quang đã chú trọng đầu tư phát triển một số môn Thể thao mũi nhọn, trong đó không thể không nhắc đến môn Wushu. Tuy nhiên, thành tích môn này chưa giành được thứ hạng cao ở các giải thi đấu lớn.

Thực tiễn cho thấy, nam VĐV Wushu lứa tuổi 1314 của tỉnh khi tham gia thi đấu ở các giải có kỹ thuật tương đối tốt, song thể lực các VĐV chưa đáp ứng được yêu cầu của giải đấu. Một trong những nhiệm vụ quan trọng của các huấn luyện viên (HLV) là phải nâng cao hiệu quả huấn luyện thể lực cho nam VĐV. Đây là nhiệm vụ hết sức khó khăn đòi hỏi nhiều công sức, trí tuệ và điều kiện cơ sở vật chất. Vì vậy, lựa chọn các biện pháp nâng cao hiệu quả huấn luyện thể lực cho nam VĐV Wushu lứa tuổi 13-14 tỉnh Tuyên Quang là vấn đề mang tính cấp thiết.
Quá trình nghiên cứu sử dụng các phương pháp: Tổng hợp và phân tích tài liệu, phỏng vấn bằng phiếu hỏi, kiểm tra sư phạm, phương pháp quan sát sư phạm, thực nghiệm sư phạm, toán học thống kê.

\section{Nội dung nghiên cứu}

2.1. Lụa chọn biện pháp nâng cao hiệu quả huấn luyện thể lục chuyên môn của nam VĐV Wushu lứa tuổi 13-14

Quá trình nghiên cứu các tài liệu đề tài đã lựa chọn được 3 nhóm biện pháp nâng cao hiệu quả huấn luyện thể lực chuyên môn (TLCM) của nam VĐV Wushu lứa tuổi 13-14 của tỉnh, đó là: - Nhóm biện pháp về chuyên môn (bài tập, lượng vận động (LVĐ), phương pháp tập luyện, thời điểm huấn luyện TLCM); - Nhóm biện pháp về trang thiết bị và dụng cụ tập luyện; - Nhóm biện pháp về tổ chức và quản lý quá trình huấn luyện.

Để có được các biện pháp nâng cao hiệu quả huấn luyện TLCM của nam VĐV Wushu lứa tuổi 13-14 mang tính khoa học, chúng tôi đã tiến hành phỏng vấn các cán bộ quản lí về TDTT, các HLV, giáo viên TDTT bằng phiếu hỏi. Số phiếu phát ra 25 , thu về 25. 
Cách trả lời cụ thể:

Ưu tiên 1: 3 điểm

Ưu tiên 2: 2 điểm

Uu tiên 3: 1 điểm

U’u tiên 4: 0 điểm

\begin{tabular}{|c|c|c|c|c|c|c|c|c|c|c|c|}
\hline \multirow{3}{*}{ TT } & \multirow{3}{*}{ Tên nhóm biện pháp } & \multicolumn{10}{|c|}{ Kết quả phỏng vấn } \\
\hline & & \multicolumn{2}{|c|}{ Ưu tiên 1} & \multicolumn{2}{|c|}{$\begin{array}{l}\text { Uu tiên } \\
2\end{array}$} & \multicolumn{2}{|c|}{$\begin{array}{l}\text { Ưu tiên } \\
3\end{array}$} & \multicolumn{2}{|c|}{\begin{tabular}{|c} 
U'u tiên \\
4
\end{tabular}} & \multirow[t]{2}{*}{\begin{tabular}{|l|} 
Tồng \\
diếm
\end{tabular}} & \multirow[t]{2}{*}{$\%$} \\
\hline & & $\mathbf{n}$ & a & $\mathbf{n}$ & a & $\mathbf{n}$ & a & $\mathbf{n}$ & a & & \\
\hline 1 & $\begin{array}{l}\text { Nhóm biện pháp về } \\
\text { chuyên môn (bài tập, LVĐ, } \\
\text { phương pháp tập luyện, thời } \\
\text { điểm huấn luyện TLCM). }\end{array}$ & 22 & 66 & 3 & 6 & 0 & 0 & 0 & 0 & 72 & 96.0 \\
\hline 2 & $\begin{array}{l}\text { Nhóm biện pháp về trang } \\
\text { thiết bị và dụng cụ tập luyện. }\end{array}$ & 20 & 60 & 3 & 6 & 2 & 1 & 0 & 0 & 67 & 85.7 \\
\hline 3 & $\begin{array}{l}\text { Nhóm biện pháp về tổ } \\
\text { chức và quản lý quá trình } \\
\text { huấn luyện. }\end{array}$ & 21 & 63 & 3 & 6 & 1 & 1 & 0 & 0 & 70 & 93.3 \\
\hline
\end{tabular}

Bảng 1: Bảng tổng hợp kết quả phỏng vấn lựa chọn biện pháp nâng cao hiệu quả huấn luyện TLCM của nam VĐV Wushu lúa tuổi 13-14

Từ số liệu bảng 1 cho thấy, cả 3 nhóm biện pháp do chúng tôi đề xuất đều được các chuyên gia tán thành với kết quả đồng ý đạt từ 85.7\% - 96.0\% tổng điểm tối đa. Như vậy, có thể cho phép chúng tôi lựa chọn 3 nhóm biện pháp với các nội dung của từng biện pháp như sau:

-Nhóm biện pháp về chuyên môn

+ Mục đích:

Nhằm nâng cao hiệu quả huấn luyện thể lực cho nam VĐV Wushu lứa tuổi 13-14 tỉnh Tuyên Quang.

+ Nội dung:

Bao gồm 12 bài tập: + Đá vòng cầu vào đích cố định 30s (lần); + Tại chỗ đá ngang vào đích cố định $30 \mathrm{~s}$ (lần); + Đá tống sau vào đích cố định 30 s (lần); + Di chuyển đá vòng cầu và đá chẻ thượng đẳng 30 s (lần); + Di chuyển đá vòng cầu (đá kẹp) 2 chân vào đích $30 \mathrm{~s}$

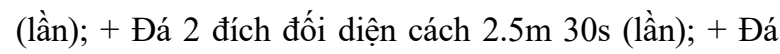
trước hai chân liên tục vào đích trong 1 phút (số lần); + Gánh tạ $15 \mathrm{~kg}$, lướt đá ngang sang hai bên trong 1 phút (số lần); + Đeo bao chì $5 \mathrm{~kg}$, đá trước liên tục trong 1 phút (số lần); + Đá thẳng, tính khoảng cách mũi chân đá tới so với đỉnh đầu; + Đá vòng cầu kết hợp đá vòng sau và lướt đá ngang vào đích trong 30 giây (số lần); + Một chân đá trước, vòng cầu và đá chẻ liên tục trong 30 giây (số lần).

\section{+ Tổ chức thực hiện}

Thời gian thực hiện là 03 tháng nằm trong giai đoạn III (Giai đoạn hoàn thiện thể lực chung, chuyên môn, tiếp tục hoàn thiện kỹ chiến thuật cho VĐV) của kế hoạch huấn luyện năm.

Số buổi tập là 3 buổi/tuần; thời gian tập 30 phút/buổi; các bài tập được thực hiện ở cuối các buổi tập sau nội dung tập kỹ thuật và chiến thuật.

$+V \hat{\hat{e}} L V Ð$ :

Cường độ Xác định cường độ vận động thông qua tần số mạch của các $\mathrm{VĐV}$, luôn duy trì tần số mạch trong phạm vi 180 lần/1 phút trở xuống.

Khối lượng: 3 tổ/1 bài tập

Quãng nghỉ: Huấn luyện viên chủ yếu sử dụng phương pháp nghỉ ngơi tích cực.

+ Về phuoong pháp tập luyện

Các phương pháp trong huấn luyện thể lực cho nam VĐV Wushu lứa tuổi 13-14 cần tuân thủ phương pháp tập luyện có định mức chặt chẽ.

- Nhóm biện pháp về trang thiết bị và dụng cu tập luyện

+ Mục đích: Hỗ trợ cho việc thực hiện tập luyện các bài tập trong nhóm biện pháp chuyên môn nhằm nâng cao hiệu quả huấn luyện thể lực cho nam VĐV Wushu lứa tuổi 13-14 tỉnh Tuyên Quang.

+ Nội dung: Đề xuất, yêu cầu Trung tâm TDTT tỉnh bổ sung, tăng cường các trang thiết bị dụng cụ thiết yếu phục vụ tập luyện của VĐV gồm:

\begin{tabular}{|c|l|c|}
\hline STT & \multicolumn{1}{|c|}{ Tên dụng cụ } & Số lượng \\
\hline 1 & Bao cát & 10 bao \\
\hline 2 & Tạ & 5 bộ \\
\hline 3 & Lăm bơ & 20 chiếc \\
\hline 4 & Giầy tập & 20 đôi \\
\hline 5 & Trang phục tập luyện & 20 bộ \\
\hline 6 & Mờ rộng diện tích phòng tập & $100 \mathrm{~m}^{2}$ \\
\hline
\end{tabular}

+ Tổ chức thực hiện: Ban huấn luyện lập đề xuất yêu cầu bổ sung tăng cường các trang thiết bị dụng cụ thiết yếu phục vụ tập luyện của VĐV lên lãnh đạo Trung tâm TDTT Tỉnh để phê duyệt.

- Nhóm biện pháp về tổ chức và quản lý quá trình huấn luyện

+ Mục đích: Hỗ trợ cho việc huấn luyện đạt hiệu quả cao

+ Nội dung:

Về quản lý: Trong sinh hoạt đảm bảo thực hiện đúng theo nội quy, quy định về thời gian, ăn, ngủ, học văn hóa của Ban huấn luyện, Ban quản lý VĐV tại Trung 
tâm Huấn luyện TDTT tỉnh đề ra. Trong tập luyện cần đảm bảo an toàn, thực hiện đúng giáo trình, giáo án buổi tập, thực hiện theo sự chỉ dẫn của các HLV.

Về tổ chức huấn luyện: Đảm bảo theo cấu trúc của huấn luyện thể thao là một trật tự liên kết tương đối ổn định của những thành phần, nội dung, các khâu trong đó. Cấu trúc buổi tập của nam VĐV Wushu lứa tuổi 1314 theo một trật tự nhất định. Buổi tập gồm 3 phần: chuẩn bị (khởi động), cơ bản và kết thúc. Các bài tập nâng cao thể lực được đưa vào trong phần cuối của phần cơ bản sau khi đã luyện tập xong phần kỹ thuật, chiến thuật.

+ Tổ chức thực hiện

Quản lý nội vụ: được giao cho phòng hành chính của Trung tâm TDTT tỉnh Tuyên Quang.

Quản lý huấn luyện: được giao cho Ban huấn luyện của đội tuyển Wushu của Trung tâm TDTT tỉnh Tuyên Quang.

2.2. Úng dụng các nhóm biện pháp nhằm nâng cao hiệu quả huấn luyện TLCM của nam VĐV Wushu lứa tuổi 13-14

\subsubsection{Tổ chức ứng dụng}

Thờ gian thực nghiệm: 03 tháng (từ tháng 8 đến tháng 11 năm 2018).

Địa điểm thục nghiệm: Trung tâm huấn luyện thể thao tỉnh.

Đối tuợng thực nghiệm: 20 nam VĐV Wushu lứa tuổi 13-14 tỉnh Tuyên Quang, được chia làm hai nhóm: Nhóm thực nghiệm và nhóm đối chứng, mỗi nhóm gồm $10 \mathrm{VĐV}$ được tiến hành trên cùng một địa điểm cùng HLV, với 02 phòng tập sát cạnh nhau, nội dung tập luyện để phát triển TLCM của hai nhóm khác nhau:

- 10 VĐV nhóm thực nghiệm tập các nhóm biện pháp do chúng tôi lựa chọn.

- 10 VĐV nhóm đối chứng tập theo giáo án chung.

\subsection{Thực nghiệm đánh giá các biện pháp nâng cao hiệu quả huấn luyện TLCM của nam VĐV Wushu lứa tuổi 13-14}

\subsection{1. Đánh giá kết quả các nhóm biện pháp}

Từ các bước nghiên cứu trên chúng tôi tiến hành triển khai thực nghiệm các nhóm biện pháp và đánh giá hiệu quả các nhóm biện pháp này trong thực tiễn huấn luyện nam VĐV Wushu lứa tuổi 13-14 ở tỉnh.
Trước khi đánh giá kết quả trình độ TLCM của nam VĐV Wushu lứa tuổi 13-14, đề tài đánh giá kết quả các biện pháp đã đề xuất:

- Nhóm biện pháp về chuyên môn

Quá trình kiểm tra, giám sát các buổi tập luyện chúng tôi nhận thấy các $H L V$ và VĐV Wushu lứa tuổi 13-14 ở tỉnh đã thực hiện đúng các quy trình, đúng giáo án đề ra cho từng buổi tập. Các HLV và VĐV đều có nhận xét về các bài tập và khối lượng vận động của các bài tập, buổi tập là vừa sức với trình độ của VĐV. 100\% VĐV đều hoàn thành tốt khối lượng các bài tập trong từng buổi tập.

-Nhóm biện pháp về trang thiết bị và dụng cu tập luyện

Tuyên Quang là một tỉnh miền núi, điều kiện phát triển kinh tế - xã hội còn nhiều khó khăn, do đó, để đầu tư kinh phí, mua trang thiết bị phục vụ nhu cầu tập luyện cho VĐV Wushu còn rất hạn chế. Tuy nhiên, được sự quan tâm của lãnh đạo Sở Văn hóa - Thể thao và Du lịch tỉnh cũng như lãnh đạo Trung tâm Huấn luyện TDTT về một số đề xuất, kiến nghị mua thêm trang thiết bị dụng cụ tập luyện cho VĐV đội tuyển Wushu tỉnh, Ban huấn luyện đội tuyển đã được Ban lãnh đạo Sở chấp thuận, đồng ý trang bị cho đội tuyển Wushu gồm các dụng cụ sau: 6 bao cát; 3 bộ tạ; 15 lăm pơ; cấp phát cho mỗi VĐV 1 bộ quần áo đồng phục Wushu, 1 đôi giày chạy; Diện tích phòng tập dành cho môn Wushu được bố trí rộng hơn khoảng hơn $100 \mathrm{~m}^{2}$ trong nhà thi đấu của Trung tâm Huấn luyện TDTT tỉnh.

-Tổ chức và quản lý quá trình huấn luyện

Sau khi áp dụng nhóm biện pháp này các VĐV đã nâng cao được ý thức tổ chức kỷ luật trong sinh hoạt cũng như tập luyện. Về sinh hoạt các em sống nề nếp, luôn thực hiện đúng giờ quy định. Về tổ chức huấn luyện các em hăng say tập luyện và luôn nêu cao tinh thần, ý chí vượt qua khó khăn trong điều kiện tập luyện còn thiếu thốn.

2.3.2. Đánh giá trình độ phát triển TLCM của nam V円V Wushu lứa tuổi 13-14 sau khi áp dụng các nhóm biện pháp

Trên cơ sở đánh giá thực trạng trình độ thể lực của nam VĐV Wushu lứa tuổi 13-14, đề tài tiến hành đánh giá hiệu quả các nhóm biện pháp sau 3 tháng ứng dụng. Các chỉ tiêu kiểm tra, đánh giá được lựa đó là:

Súc manh: - Đấm thẳng hai tay tốc độ vào lăm pơ $15 \mathrm{~s}$ (lần); - Đá vòng cầu hai chân vào lăm pơ $15 \mathrm{~s}$ (lần). 
Súc nhanh: - Đá vòng cầu chân thuận vào bao 30s (lần); - Quét trước, quét sau có đích 30s (lần).

Sức bền: - Đá vòng cầu hai chân vào lăm pơ $120 \mathrm{~s}$ (lần); - Di chuyển ra tổ hợp đòn 120s (tổ).

Khả năng phối hợp vận động: - Đá vòng cầu rút chân đá về quét sau 60 s (lần); - Kết hợp đấm thẳng-đá vòng cầu hai chân liên tục 30s (lần).

Bảng 2: So sánh sụ phát triển TLCM của nam VĐV Wushu lứa tuổi 13 trước và sau khi áp dụng các nhóm biện pháp

\begin{tabular}{|c|c|c|c|c|c|}
\hline \multirow[b]{2}{*}{ TT } & \multirow[b]{2}{*}{ Test } & \multicolumn{3}{|c|}{ Lứa tuối 13} & \multirow[b]{2}{*}{$\mathbf{P}$} \\
\hline & & $\begin{array}{c}\text { Trước thực } \\
\text { nghiệm } \\
\bar{X} \pm \delta\end{array}$ & $\begin{array}{l}\text { Sau thực nghiệm } \\
\qquad \bar{X} \pm \delta\end{array}$ & $\mathrm{t}$ & \\
\hline 1 & $\begin{array}{l}\text { Đấm thẳng hai tay tốc độ } \\
\text { vào lăm pơ } 15 \mathrm{~s} \text { (lần) }\end{array}$ & $28.75 \pm 3.25$ & $31.9 \pm 4.01$ & 3.425 & $<0.05$ \\
\hline 2 & $\begin{array}{l}\text { Đá vòng cầu hai chân vào } \\
\text { lăm po } 15 \mathrm{~s} \text { (lần) }\end{array}$ & $24.8 \pm 3.52$ & $31.5 \pm 3.44$ & 4.335 & $<0.05$ \\
\hline 3 & $\begin{array}{l}\text { Đá vòng cầu chân thuận vào } \\
\text { bao } 30 \text { s (lần) }\end{array}$ & $31.9 \pm 3.15$ & $34.7 \pm 4.12$ & 3.363 & $<0.05$ \\
\hline 4 & $\begin{array}{l}\text { Quét trước, quét sau có đích } \\
\text { 30s (lần) }\end{array}$ & $21.4 \pm 3.24$ & $25.6 \pm 2.8$ & 4.492 & $<0.05$ \\
\hline 5 & $\begin{array}{l}\text { Đá vòng cầu hai chân vào } \\
\text { lăm po } 120 \text { s (lần) }\end{array}$ & $100.2 \pm 24.24$ & $121.2 \pm 21.5$ & 10.716 & $<0.01$ \\
\hline 6 & $\begin{array}{l}\text { Di chuyền ra tồ hợ đòn } \\
120 \text { s (tồ) }\end{array}$ & $15.4 \pm 4.42$ & $18.2 \pm 3.47$ & 2.382 & $<0.05$ \\
\hline 7 & $\begin{array}{l}\text { Đá vòng cầu rút chân đá về } \\
\text { quét sau } 60 \text { s(lần) }\end{array}$ & $18.4 \pm 1.83$ & $21.9 \pm 2.27$ & 5.230 & $<0.05$ \\
\hline 8 & $\begin{array}{l}\text { Kêt hợp đấm thẳng - đả } \\
\text { vòng cầu hai chân liên tục }\end{array}$ & $11.5 \pm 3.12$ & $13.9 \pm 2.21$ & 2.157 & $<0.05$ \\
\hline
\end{tabular}

Bảng 3: So sánh sụ phát triển TLCM của nam VĐV Wushu lứa tuổi 14 trước và sau khi áp dụng các nhóm biện pháp

\begin{tabular}{|c|c|c|c|c|c|}
\hline \multirow[b]{2}{*}{ TT } & \multirow[b]{2}{*}{ Test } & \multicolumn{3}{|c|}{ Lúa tuổi 13} & \multirow[b]{2}{*}{$\mathbf{P}$} \\
\hline & & $\begin{array}{c}\text { Trước thục } \\
\text { ngliệm } \\
\bar{X} \pm \delta\end{array}$ & $\begin{array}{c}\text { Sau thục nghiệm } \\
\bar{X} \pm \delta\end{array}$ & t & \\
\hline 1 & $\begin{array}{l}\text { Đấm thẳng hai tay tốc độ } \\
\text { vào lăm pơ } 15 \mathrm{~s} \text { (lần) }\end{array}$ & $28.75 \pm 3.25$ & $31.9 \pm 4.01$ & 3.425 & $<0.05$ \\
\hline 2 & $\begin{array}{l}\text { Đá vòng cầu hai chân vào } \\
\text { lăm pơ } 15 \mathrm{~s} \text { (lần) }\end{array}$ & $24.8 \pm 3.52$ & $31.5 \pm 3.44$ & 4.335 & $<0.05$ \\
\hline 3 & $\begin{array}{l}\text { Đá vòng cầu chân thuận vào } \\
\text { bao } 30 \text { s (lần) }\end{array}$ & $31.9 \pm 3.15$ & $34.7 \pm 4.12$ & 3.363 & $<0.05$ \\
\hline 4 & $\begin{array}{l}\text { Quét trước, quét sau có đích } \\
\text { 30s (lần) }\end{array}$ & $21.4 \pm 3.24$ & $25.6 \pm 2.8$ & 4.492 & $<0.05$ \\
\hline 5 & $\begin{array}{l}\text { Đá vòng cầu hai chàn vào } \\
\text { lăm pơ } 120 \text { s (lần) }\end{array}$ & $100.2 \pm 24.24$ & $121.2 \pm 21.5$ & 10.716 & $<0.01$ \\
\hline 6 & $\begin{array}{l}\text { Di chuyền ra tồ họp đòn } \\
120 \text { s (tồ) }\end{array}$ & $15.4 \pm 4.42$ & $18.2 \pm 3.47$ & 2.382 & $<0.05$ \\
\hline 7 & $\begin{array}{l}\text { Đá vòng cầu rút chân đá về } \\
\text { quét sau } 60 \text { s(lần) }\end{array}$ & $18.4 \pm 1.83$ & $21.9 \pm 2.27$ & 5.230 & $<0.05$ \\
\hline 8 & $\begin{array}{l}\text { Kết hợp đẩm thẳng - đá } \\
\text { vòng cầu hai chân liên tục }\end{array}$ & $11.5 \pm 3.12$ & $13.9 \pm 2.21$ & 2.157 & $<0.05$ \\
\hline
\end{tabular}

Qua kết quả bảng 2 và 3 cho thấy, sau 3 tháng ứng dụng các nhóm biện pháp trình độ TLCM của nam VĐV Wushu lứa tuổi 13-14 sau thực nghiêm so với trước thực nghiệm tăng lên đáng kể $(\mathrm{P}<0.05$ đến , 0.01) ở cả 2 nhóm lứa tuổi 13-14, nhất là nội dung đá vòng cầu hai chân vào lăm pơ 120 s (lần) của cả 2 lứa tuổi 1314 đều có sự tăng trưởng rõ rệt $(\mathrm{P}<0.01)$.

Như vậy, có thể kết luận, trình độ TLCM của nam VĐV Wushu lứa tuổi 13-14 sau 3 tháng thực nghiệm đã có sự tăng trưởng rõ rệt. Để đánh giá chính xác hiệu quả của các nhóm biện pháp, chúng tôi đã tiến hành kiểm tra và phân tích nhịp độ tăng trưởng về TLCM của nam VĐV Wushu lứa tuổi 13-14 trước và sau thực nghiệm. Kết quả được trình bày ở bảng 4 .

Bảng 4: Nhịp tăng truởng TLCM của nam VĐV Wushu lứa tuổi 13-14

\begin{tabular}{|c|c|c|c|c|c|}
\hline \multirow{2}{*}{ TT } & \multirow{2}{*}{ Test } & \multicolumn{2}{|c|}{$\begin{array}{c}\text { W (\%) } \\
\text { Lúa tuổi } 13\end{array}$} & \multicolumn{2}{|c|}{$\begin{array}{l}\text { W (\%) } \\
\text { Lúa tuổi }\end{array}$} \\
\hline & & $\begin{array}{c}\text { Trưóc } \\
\text { TN }\end{array}$ & $\begin{array}{l}\text { Sau } \\
\text { TN }\end{array}$ & $\begin{array}{c}\text { Trước } \\
\text { TN }\end{array}$ & $\begin{array}{l}\text { Sau } \\
\text { TN }\end{array}$ \\
\hline 1 & $\begin{array}{l}\text { Đấm thẳng hai tay tốc độ vào lăm pơ } 15 \mathrm{~s} \\
\text { (lần) }\end{array}$ & 8.21 & 9.71 & 8.73 & 10.72 \\
\hline 2 & Đá vòng cầu hai chân vào lăm pơ $15 \mathrm{~s}$ (lần) & 9.12 & 12.44 & 9.25 & 13.7 \\
\hline 3 & Đá vòng cầu chân thuận vào bao 30s (lần) & 6.53 & 8.42 & 7.03 & 8.54 \\
\hline 4 & Quét trước, quét sau có đích 30s (lần) & 10.54 & 13.09 & 11.01 & 13.33 \\
\hline 5 & Đá vòng cầu hai chân vào lăm pơ $120 \mathrm{~s}$ (lần) & 13.25 & 19.23 & 15.34 & 21.94 \\
\hline 6 & Di chuyền ra tổ hợp đòn 120s (tổ) & 8.64 & 11.84 & 8.89 & 13.18 \\
\hline 7 & Đá vòng cầu rút chân đá về quét sau 60s(lần) & 10.37 & 14.61 & 10.87 & 17.56 \\
\hline 8 & $\begin{array}{l}\text { Kết hợp đầm thẳng - đá vòng cầu hai chân } \\
\text { liên tục 30s (lần) }\end{array}$ & 6.21 & 8.33 & 7.12 & 9.33 \\
\hline
\end{tabular}

Qua bảng 4 cho thấy, sau 3 tháng thực nghiệm, nhịp độ tăng trưởng về trình độ TLCM của nam VĐV Wushu lứa tuổi 13-14 của tỉnh Tuyên Quang ở mức tốt. Nhịp độ tăng trưởng sau thực nghiệm cao hơn trước thực nghiệm từ $0.96 \%$ $6.69 \%$.

\section{Kết luận}

Từ kết quả nghiên cứu đề tài đã lựa chọn được 3 nhóm biện pháp nhằm phát triển TLCM của nam VĐV Wushu lứa tuổi 13-14, đó là: - Nhóm biện pháp chuyên môn (gồm bài tập, LVĐ, phương pháp tập luyện, thời điểm huấn luyện TLCM); - Nhóm biện pháp tăng cường cơ sở vật chất, trang thiết bị và dụng cụ phục vụ công tác huấn luyện; - Nhóm biện pháp tổ chức và quản lý quá trình huấn luyện.

Kết quả thực nghiệm sư phạm ứng dụng các nhóm biện pháp trên trong thực tiễn huấn luyện đã chứng minh tính hiệu quả và phù hợp của các nhóm biện pháp thông qua sự phát triển rõ rệt về trình độ TLCM của nam VĐV Wushu lứa tuổi 13-14 tỉnh Tuyên Quang.

\section{TÀI LIỆU THAM KHẢO}

\section{Aulic V.I (1982), Đánh giá trình độ luyện tập thể} thao, Nxb Thể dục thể thao, Hà Nội.

2. Dương nghiệp Chí (1991), Đo lường thể thao, Nxb Thể dục thể thao, Hà Nội.

3. Dương Nghiệp Chí, Nguyễn Danh Thái (2002), Công nghệ đào tạo vận động viên trình độ cao, $\mathrm{Nxb}$ Thể dục thể thao, Hà Nội. 
4. G.Macximenco (1980), "Tố chất thể lực và thành tích”, Dịch: Nguyến Kim Minh, bản tin KHKT TDTT, 9, $\operatorname{tr} 20-23$.

5. Nguyễn Ngọc Cừ, Nguyễn Kim Minh (1999), Các phuong pháp y sinh hoc kiểm tra đánh giá luợng vận động của bài tập, Viện KHTDTT, Hà Nội.
6. Oelchlegen.G, Legơ.K (1985), Bốn nhân tố nâng cao thành tích tập luyện, $\mathrm{Nxb}$ Thể.

dục thể thao, Hà Nội.

7. V. Diatstowcop (1963), Rèn luyện thể lục của VĐV, Dịch Nguyễn Trình, Nxb TDTT, Hà Nội.

\section{Improving the effectiveness of professional fitness training of male wushu athletes at the age of 13-14 in Tuyen Quang Province}

Tran Vu Phuong, Luc Hung Quoc

\section{Article info}

Recieved:

27/11/2018

Accepted:

$10 / 12 / 2018$

Keywords:

Measures; improve

effectiveness; professional

fitness; coach; male

Wushu athletes at the age

of 13-14.

\section{Abstract}

In order to improve fitness training for male Wushu athletes at 13-14 age groups in Tuyen Quang province, the project has selected 3 groups of measures. Three groups of measures affirm the effectiveness of fitness training for male Wushu athletes at the age of 13-14 in Tuyen Quang Province. 